\title{
La interlengua en los primeros estadios de aprendizaje de una lengua extranjera (inglés)
}

\author{
M. Rosa Torras
}

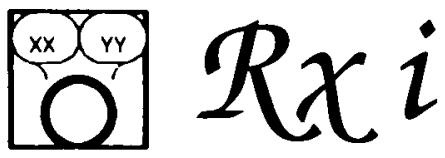

La autora aborda en este artículo una de las cuestiones candentes en la enseñanza de la lengua extranjera. ¿hasta qué punto aquello que se aprende se traduce en desarrollo lingüistico?. Este estudio sobre la interlengua de los escolares de EGB que aprenden inglés da respuestas a este interrogante.

\section{INTRODUCCION}

Un fenómeno fácilmente observable en la enseñanza de las lenguas extranjeras, pero enigmático y frustrante a la vez, es la lentitud y la falta de acoplamiento con que se aprenden. El profesor presenta repetidamente en clase unos modelos de lengua normativa, pero los alumnos experimentan grandes dificultades y oscilaciones en su incorporación. Se observa, además, que el tipo de tareas que el profesor plantea en clase influye significativamente en el nivel diferencial de corrección del uso que de la lengua hacen los alumnos. Estas caraterísticas atípicas, han llevado a comparar el proceso de aprendizaje de una lengua extranjera en el ámbito escolar al del aprendizaje de una lengua primera o segunda en el ámbito natural. Según esta concepción naturalista del proceso de adquisición de las lenguas, el aprendizaje de un idioma extranjero, no puede compararse nunca de forma satisfactoria con el de ninguna otra asignatura, sino que debe contemplarse como un proceso con peculiaridades específicas. Entre ellas cabría señalar, en primer lugar, que el sistema lingüístico que construye un alumno en los primeros estadios iniciales del proceso de adquisición de un idioma extranjero debe considerarse como un sistema aproximativo con entidad propia.

El término interlengua, introducido por primera vez por Selinker en 1972, se ha utilizado para referirse a este sistema lingüístico aproximativo, "la gramática mental ", que el aprendiz de una lengua va construyendo en el proceso de adquisición de la misma. Este sistema o interlengua no puede considerarse como una versión errónea de la lengua que se aprende, sino como un sistema en sí mismo. El concepto implica que el sistema lingüístico del aprendiz no es ni el de la L1 ni el de la L2 aunque contenga elementos de ambas. Los resultados de diversos estudios empíricos llevan a concluir que los aprendices de una lengua avanzan a lo largo de un pro- 
ceso, urilizando estas gramáticas provisionales que se van desarrollando y enriqueciendo a través de una serie de estadios transicionales en el camino hacia la adquisición de la lengua objeto de aprendizaje.

\section{EL PROCESO DE ADQUISICION DE LA LENGUA}

La teoría de la interlengua se construyó sobre los mismos principios mentalistas que explican la adquisición de la Ll : énfasis sobre la formación y verificación de hipótesis y procesos internos, así como la noción de un continuum de aprendizaje que implica sucesivas reestructuraciones progresivas del sistema interno.

Para explicar cómo el niño que aprende la primera lengua (L1) la va construyendo o desarrollando se ha utilizado el concepto de bypothesis-testing. Según esta teoría el niño va formando hipótesis a propósito de las transformaciones necesarias para convertir el conocimiento innato que tiene de unos principios gramaticales universales, presentes en todas las lenguas, en las formas superficiales propias de la lengua que está aprendiendo. Este proceso conlleva la formulación, verificación y modificación de hipótesis hasta llegar a adquirir el modelo lingüístico del adulto (Mc Neill, 1966, 1970).

De acuerdo con esta teoría el aprendiz de una segunda lengua o de una lengua extranjera formula también, de forma consciente o inconsciente, hipótesis sobre las reglas de la L2 con el fin de confirmarlas o abandonarlas y, en este último caso, sustituirlas por hipótesis nuevas. Dos son las posiciones sostenidas por los autores respecto a la base de la que parten los aprendices en la construcción de sus hipótesis: para unos (Burt \& Dulay, 1977; Corder, 1978) los aprendices parten de una gramática innata al igual que el niño pequeño con la que van construyendo el sistema interlingüístico (recreating continuum); para otros (Nemser, 1971) el punto de partida es la L1 de la que van substituyendo gradualmente sus características por las de la L2 (restructuring continuum). La mayoría de teóricos hoy en día asumen la hipótesis del recreating continuum aunque sin excluir la posibilidad de transferencia entre la primera $(\mathrm{L} 1)$ y la segunda lenguas (L2).

Sin embargo, y a diferencia de los aprendices de una $L 1$, los aprendices de una L2/LE pueden quedarse a medio camino del proceso y no llegar a desarrollar por completo el sistema lingüístico de la lengua objeto de aprendizaje, «fosilizando» su sistema lingüístico en un estadio determinado (Selinker, 1972).

Otra cuestión a tener en cuenta, y que es de gran interés para el profesor, es saber en qué condiciones manifiestan los aprendices esta interlengua. Según varios autores (Tarone, 1983; Ellis, 1985) la interlengua se activa especialmente en situaciones de la producción lingüística creativa y espontánea, mientras que, en condiciones de elevado control ambiental su actuación se ve parcialmente inhibida.

Una observación atenta de los libros de texto, correspondientes a los primeros niveles de enseñanza, pone de manifiesto, sin embargo, que la mayoría de las actividades propuestas para la clase son controladas, ya que predeterminan el lenguaje que el aprendiz ha de usar, ofreciendo los modelos lingüísticos que se han de utilizar en el desarrollo de la actividad escolar. Por el contrario son muy pocas las actividades propuestas que permiten al alumno poner en acto de manera libre y espontánea los recursos lingüísticos de que dispone. La actividad siguiente, tomada de un libro de texto, es un ejemplo representativo de esta situación de control en que los alumnos se ven obligados a formular sus preguntas y respuestas siguiendo los modelos suministrados (ver Figura 1).

No se puede decir, desde el punto de vista lingüístico, que este tipo de actividad sea creativa, ya que los alumnos se ven constreñidos a utilizar el modelo suministrado. Su finalidad es la de permitir a los alumnos practicar determinadas estruc- 
turas sin cometer errores. En el marco de estas actividades controladas el alumno es capaz de formular preguntas y dar respuestas emitiendo los componentes de la frase en el orden adecuado según correspondan a oraciones interrogativas o declarativas. Estos resultados podrían hacer creer al profesor que el alumno ha incorporado las reglas del orden de las palabras de la interrogación, pero esto sólo podría comprobarse si el alumno fuera también capaz de formular preguntas correctas en situaciones de activación libre de las reglas supuestamente interiorizadas.

FIGURA 1

(Ejemplo de actividad didáctica. Reproducido de Hutchison, 1985, p. 67)

How many legs have these animals got?

Have they got any arms?

$\begin{array}{lll}\text { monkey } & \text { dog } & \text { lion } \\ \text { bird } & \text { snake } & \text { gorilla } \\ \text { spider } & \text { fish } & \text { cat } \\ \text { man } & \text { insect } & \text { rat }\end{array}$

Ask and answer like this:

A.- How many legs bas a monkey got?

B.-It's got two legs

A.- Has it got any arms?

B.-Yes. It's got two arms.

Una actividad didáctica en la que se estimula el uso espontáneo de la lengua es, por ejemplo, la de llevar a cabo una entrevista, poniendo al alumno en condiciones de utilizar sus propios recursos lingüísticos en la lengua que está aprendiendo. Las preguntas que ofrecemos a continuación fueron formuladas por un alumno de $8^{\circ}$ de EGB mientras entrevistaba a un profesor de su escuela (ver Figura 2).

FIGURA 2

Emisiones lingü̈ísticas producidas por un alumno de $8^{\circ}$ de E.G.B.

\begin{tabular}{|l|}
\hline 1-what's your name? \\
2-bow old are you? \\
3-do you like English? \\
4-bave you brothers and sisters? \\
5-bow old are your sister? \\
6-you play football? \\
7-you study a French? \\
8-you are a teacher?
\end{tabular}

El alumno empieza utilizando cuatro preguntas formalmente correctas, en las que aplica adecuadamente las reglas de interrogación tanto en preguntas Wh-, que inician con pronombres interrogativos, como en preguntas Yes/No, que exigen respuestas afirmativas o negativas. Sería demasiado optimista, sin embargo, suponer que el alumno domina las reglas de la interrogación, ya que las preguntas siguientes, de la 5 a la 8 , presentan desviaciones formales que evidencian su falta de dominio formal del sistema lingüístico objeto de aprendizaje. Ello nos lleva a sospechar que las cuatro primeras preguntas son la reproducción de frases o fórmulas memorizadas; 
cuando el alumno, en cambio, agota los recursos memorizados (que no por ello dejan de cumplir una función comunicativa) construye sus preguntas en base a sus propias hipótesis. La pregunta 5, por ejemplo, parece estar construida en base a la rutina memorizada de la pregunta 2 aunque gramaticalmente sea incorrecta; las preguntas 6,7 y 8 reflejan las primeras fases de la adquisición de la interrogación en la que los aprendices utilizan el orden declarativo, omitiendo el auxiliar y dejando que sea la entonación la que indique la función interrogativa.

La observación sistemática de estos fenómenos (Torras, 1992) pone de manifiesto que cuando se permite al aprendiz usar con libertad sus recursos lingüísticos, éste puede producir emisiones lingüísticas que no se ajustan a los modelos practicados en clase, y que constituyen una manifestación de su interlengua.

En esta situación el profesor puede experimentar una sensación de frustración, ya que la producción del alumno presenta desviaciones respecto al sistema linguiístico que él ha estado «enseñando» en clase. Tal frustración, sin embargo, sólo estaría justificada si el curso de aprendizaje de una lengua siguiera como un calco al del proceso de su enseñanza. Pero ello no es así. El proceso de adquisición de un lengua sigue, en efecto, una serie de estadios consecutivos, característicos de la interlengua, cuyas peculiaridades se describen a continuación, acompañadas de abundantes ejemplos de producciones lingüísticas espontáneas de aprendices de Inglés durante los primeros estadios de adquisición, en concreto por parte de alumnos de E.G.B.

\section{CARACTERISTICAS DE LA INTERIENGUA}

Como características de la interlengua suelen señalarse las siguientes:
a) Simplificación
b) Sistematicidad
c) Dinamicismo
d) Fosilización
e) Variabilidad

\section{Simplificación}

La interlengua es un sistema simplificado o reducido si se compara al sistema de la lengua que se está aprendiendo, tanto por lo que se refiere a la complejidad de las reglas morfosintácticas y pragmáticas como a la amplitud y precisión del léxico. Los aprendices simplifican porque necesitan reducir la complejidad de las operaciones que deben realizar, limitando el número de hipótesis sobre el sistema lingüístico a aquellas que son fáciles de formar y que posibilitan la comunicación.

En los primeros estadios de adquisición el aprendiz construye su discurso en base a oraciones simples utilizando al mínimo la oración compuesta. En el siguiente ejemplo se puede observar cómo un alumno construye una narración a través de una secuencia de oraciones simples dejando que sea el contexto gráfico secuencial de la historieta (ver Anexo) el que establezca las relaciones entre los hechos (ver Figura 3)

FIGURA 3

Emisiones lingüisticas producidas por alumnos de $8 .^{\circ}$ de E.G.B.
1) this children are preparing a the food/
2) bere they are they are see the map / the dog tries to to to eat the food /
3 y 4) the children are going to the country/
5) bere they are going to take the food / the dog leave for the basket I
6) there isn't the food/ the dog bas eat the food 
Este mismo ejemplo muestra otra característica que se da en los primeros estadios: los aprendices se aferran al orden rígido Sujeto + Verbo + Objeto o Complemento en sus oraciones que sólo pueden expandir con la colocación de adverbios o frases adverbiales al principio o final de la oración:

(Alumno) A: in winter sometimes I read music

Este orden también se aplica a aquellas oraciones que necesitan una reordenación de los elementos constituyentes como son las oraciones interrogativas dejando que sea la entonación ascendente la que marque la intención interrogativa.

A: the little girl study in the school (?)

A: you travel sometimes (?)

A: why why you work work in that thing (?)

También se puede dar una reducción de la proposición por omisión de alguno(s) de los constituyentes obligatorios, cuyo contenido informativo puede ser inferido por el receptor a través del contexto:

(Profesor) P: what is mother doing?

A: cut (omisión del sujeto y objeto)

(Contexto: Viñeta 1. Ver Ánexo)

Es frecuente que el aprendiz omita constituyentes con un contenido informativo poco importante como ocurre con la cópula, las formas existenciales o sujetos pronominales.

A: the tree no in the picture (omision de there is)

A: she in bouse (omisión de is)

A: they want to go to the trees because bot (omisión de it is)

Estas simplificaciones que se dan a nivel de estructura sintáctica, también se producen a nivel morfológico y/o a través de la omisión de palabras funcionales (verbos auxiliares, preposiciones, artículos etc.) y sufijos (-ed, -ing, -s, etc). Es conveniente señalar que el aprendiz conoce a veces estas formas y puede utilizarlas en sus producciones verbales, incluso de manera errónea, así como evitarlas en otras ocasiones. Ello se explicaría por la necesidad que tiene el alumno de simplificar las operaciones de procesamiento lingüístico, lo que le lleva a omitir elementos lingüísticos de menor carga informativa o que en ocasiones pueden resultar redundantes, como muestran los siguiente ejemplos:

A: two girls not not playing (omisión are)

A: the dog is eat the sandwich (omisión -ing)

P: what did you do yesterday?

A: I walk for Pineda (omisión -ed)

A: be eat a breakfast (omisión -s)

Respecto al vocabulario es obvio que en los primeros estadios de adquisición de una lengua los aprendices no disponen de suficientes recursos lexicales para llenar de contenido sus mensajes y en consecuencia manifiestan una notable pobreza de vocabulario en su interlengua. La actuación de los aprendices ante esta limitación puede presentar diferencias individuales que pueden ir desde la reducción total del mensaje o limitación del contenido del mismo al uso de estrategias comunicativas que les permitan llenar los vacíos con los recursos lingüísticos que poseen ya sea de la L1 o L2. Así por ejemplo ante la pregunta:

what did you do yesterday? 


\section{4}

un alumno adopta la estrategia de reducir el contenido a los movimientos de ida y vuelta a casa y a la escuela que puede expresar con sus limitados recursos-lexicales (ver Figura 4).

FIGURA 4

Producción oral de un alumno de $8 .^{\circ}$ de E.G.B.

A: eight o'clock went to schooll and nine o'clock go in in the schooll and balf past one go in my bomel at three o'clock go in school/ and balf past half past five go my bouse.

En otras ocasiones el alumno recurre a su L1, adaptando palabras de la misma a la fonética de la L2:

$\mathrm{P}$ : and where is the breakfast now?

A: in the paunch of the dog. (L1 Catalán panxa — panza o barriga, en castellano-)

o simplemente inserta vocabulario de su L1 en una secuencia de la L2 sin ninguna adaptación

\section{A: Jobn and Mary go to the "valle"}

\section{Sistematicidad}

Que la interlengua es sistemática (Corder, 1971; Adjemian, 1976; Selinker, 1972; Ellis, 1985) significa que constituye un sistema lingüístico en sí mismo, gobernado por reglas propias que le dan consistencia interna y que pueden ser distintas de las de la lengua que se está aprendiendo.

En los ejemplos que veremos a continuación tres alumnos, a pesar de haber practicado abundantemente las reglas de la negación, utilizan construcciones negativas que se apartan de las reglas del Inglés. Sin embargo cada uno de ellos aplica de manera bastante sistemática sus propias reglas para la negación (ver Figura 5).

Figura 5

Emisiones negativas producidas por alumnos de $80^{\circ}$ de E.G.B.

\begin{tabular}{|} 
A1: the dogs not walk \\
the boy not swimming \\
the girl not eat an ice-cream \\
the boys not bave any boat \\
A2: this boy don't read \\
a a boys not don't play with a boat \\
the girl don't eat the ice-cream \\
A3: the ball no in the beach \\
the tree no in the picture \\
the children no is the book \\
the boy no swim
\end{tabular}

En los tres casos se utiliza la negación interna, aunque cada uno de ellos recurre a un elemento negador distinto. En ninguno de los casos, no obstante, los alumnos aplican las reglas de concordancia. 


\section{Dinamicismo}

La interlengua es un sistema dinámico en el sentido que va cambiando a medida que incorpora nuevas reglas y palabras y revisa, al mismo tiempo, las reglas existentes como resultado de las hipótesis que se van construyendo sobre la lengua objeto de aprendizaje. Cada estadio constituye una interlengua o competencia transicional. Todos los estadios en conjunto constituyen un continuum de interlenguas.

Retomando como ejemplo el tema de la negación, que ya hemos abordado en el apartado anterior, diversos estudios longitudinales sobre la adquisición de la negación en Inglés (Wode, 1980; Felix, 1981; Ellis, 1984) ofrecen un perfil de desarrollo de la adquisición de la negación que sigue unos estadios predictibles y que de manera simplificada describimos a continuación:

1) Utilización de la negación externa. El marcador de negación se coloca al principio o al final de la emisión lingüística:

not dog (Milon, 1974)

red no (Ellis, 1982)

2) El elemento negador aparece dentro de la emisión lingüística.

I not like that (Ravem, 1968)

3) La negación interna se va consolidando con la utilización adecuada del verbo auxiliar según número y persona

I can not bit the ball (Wode, 1976)

I didn't can close it (Wode, 1976)

Si analizamos de nuevo las producciones negativas de los alumnos del Cuadro 5 nos daremos cuenta fácilmente de que éstos construyen sus negaciones de acuerdo con el estadio 2, colocando un elemento negador dentro de la emisión sin utilizar todavía las reglas de la concordancia. Es de esperar que si el proceso de enseñanza/aprendizaje sigue su curso los alumnos irán incorporando de manera gradual las reglas de la negación.

Estudios realizados igualmente con aprendices de Inglés en el contexto de la clase han descrito los estadios que se siguen en la adquisición de otros subsistemas gramaticales en Inglés, como la interrogación (Felix, 1981; Ellis, 1984; Torras,1993), el sistema pronominal (Felix, 1981, Felix \& Hahn, 1984; Felix \& Simmet, 1981), las oraciones de relativo (Pavesi, 1984, 1986) o el sistema verbal (Havranek, 1988, 1989; Torras, 1992).

\section{Fosilización}

La interlengua se puede fosilizar. Con este término Selinker (1972) se refiere a la tendencia de muchos aprendices a detenerse en un punto del interlanguage continuum, dando origen a un estancamiento a partir del cual ya no se progresa. Este fenómeno se observa en algunos individuos que a pesar de vivir en el contexto donde su utiliza la lengua que se aprende como vehículo de comunicación social no la desarrollan más, posiblemente porque el estadio de adquisición alcanzado les permite satisfacer sus necesidades comunicativas.

\section{Variabilidad}

Por variabilidad se entiende el uso variable que los aprendices hacen de sus recursos lingüísticos. Según Ellis (1985) debe hablarse de dos tipos de variabilidad la sistemática y la no-sistemática.

La variabilidad sistemática se puede predecir y viene determinada por el contexto que puede ser situacional o lingüístico. 
1.- La variabilidad situacional viene determinada por factores externos como el tipo de tarea, el marco físico, el interlocutor, etc. Todos los profesores han podido observar en qué medida el tipo de tarea puede determinar la corrección lingüística. Por ejemplo cuando los alumnos dirigen la atención a la forma, como en el caso de ejercicios gramaticales, suelen cometer menos errores que cuando realizan una tarea en la que la atención se dirige al contenido, como por ejemplo en la conversación libre o la composición no dirigida. En el marco escolar y con interlocutores que tienen el estatus de profesor los aprendices suelen poner más atención en la forma, monitorizando y autocorrigiendo sus emisiones lingüísticas más que en un marco natural con interlocutores que no son profesores. Tarone (1983) propone que la interlengua del aprendiz es un continuum de estilos que varían según que el grado de atención se dirija hacia la forma o el contenido. Según esto en un extremo del continuum se daría el estilo más natural y sistemático de la interlengua (vernacle style) cuando el aprendiz dirige su atención al contenido, mientras que en el polo opuesto del continuum se encontraría el estilo cuidado (careful style) que el aprendiz utilizaría en las tarea en cuya realización se dirige toda la atención a la forma, como por ejemplo la de detectar errores gramaticales. Esta variabilidad de estilos se debe, según Tarone, al nivel de atención determinado por la naturaleza de la tarea, pero creemos que también puede deberse a otros factores como el interlocutor, el marco físico o las demandas discursivas de la tarea entre otros.

2.-El segundo tipo de variabilidad sistemática viene determinado por el contexto linguḯstico. Por ejemplo la omisión del pronombre sujeto delante de la cópula es frecuente en la tercera persona

A: is a boy

A: because is difficult

en cambio muy raramente se da en primera persona

\section{A: I am a student}

Otro ejemplo nos lo proporciona Ellis (citado por Tarone 1988) al observar como resultado de sus estudios que la forma contracta de la cópula 's se da más frecuentemente cuando el elemento precedente es un pronombre personal que cuando es un sintagma nominal.

La variabilidad no-sistemática es la que no se puede predecir y es por tanto idiosincrática del individuo. De acuerdo con Ellis (1985) en los períodos iniciales de adquisición de una lengua el aprendiz puede utilizar una misma forma para expresar más funciones de las adecuadas o bien utilizar varias formas, adecuadas o no, para realizar una determinada función.

En el caso que sigue el alumno utiliza la forma negativa isn't para cubrir la cópula, la forma existencial y la posesión independientemente de su adecuación o corrección:

A: The children isn't very bappy (aren't)

It isn't flowers on the table (there aren't) the girl isn't ice cream (basn't)

Puede observarse en este otro caso como un alumno utiliza diversas formas para expresar el pasado:

P: Tell me what you did yesterday

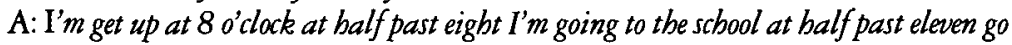
to Tordera

Esta variabilidad en el uso de las formas se da especialmente en los primeros estadios de aprendizaje tal como diversos autores han señalado, y se manifiesta espe- 
cialmente en la inestabilidad morfológica que presenta la interlengua de los aprendices. Ellis (1985) explica que esto ocurre cuando el aprendiz asimila nuevas formas pero no las integra en el sistema forma/función, es decir no sabe con certeza qué formas utilizar para qué funciones. Ellis añade que con el tiempo el aprendiz se va representando estas relaciones de acuerdo con el sistema de la lengua objeto de aprendizaje.

Es frecuente oir a profesores que expresan su desconcierto ante la actuación de los alumnos que, a pesar de los esfuerzos dedicados al estudio y práctica de determinadas formas, las utilizan de manera indiscriminada.

\section{IMPLICACIONES DIDACTICAS}

Las peculiaridades del proceso de aprendizaje de una lengua extranjera, que hemos considerado hasta este momento, son suficientes para poner de manifiesto que éste sigue un proceso particular que lo diferencia de otros aprendizajes. Este proceso pasa por una serie de estadios o fases a través de los cuales el aprendiz va construyendo de manera global la lengua que está aprendiendo. Durante este proceso de construcción el aprendiz manifiesta a través de la producción espontánea y creativa el estado de su interlengua. Esta constituye un sistema lingüístico en sí mismo que se encuentra en estado continuo de crecimiento y, en consecuencia, de fluctuación.

El aprendizaje de una lengua no sigue por tanto un proceso lineal en el que el alumno, clase tras clase, vaya incorporando los ítems lingüísticos que se le van presentando. Por el contrario, el alumno no aprende exactamente lo que se le enseña sino que de lo que se le enseña extrae en cada momento aquellos elementos que puede asimilar o integrar según el estadio de desarrollo en que se encuentra su sistema interlingüístico, el cual se pone en acto en el momento de la producción espontánea.

Independientemente de la complejidad de cualquier sistema lingüístico que se aprenda, la interlengua que se manifiesta en los primeros estadios de adquisición tiende a limitar al mínimo los recursos para expresarse, buscando la simplificación y eliminando lo que pueda ser redundante. Una estrategia utilizada por los aprendices noveles es el uso de fórmulas o de lenguaje memorizado con lo cual simplifican la complejidad que conlleva crear lenguaje. La actividad del aprendiz se limita a buscar entre sus recursos memorizados los que pueden cubrir la necesidad comunicativa del momento, evitando aplicar reglas o formular hipótesis sobre las mismas.

Ahora bien, ¿qué implicaciones didácticas se pueden derivar de todo ello para la enseñanza de la lengua extranjera y especialmente en los inicios de su enseñanza/aprendizaje en la Escuela Primaria? Si como se ha dicho el alumno no es un mero receptáculo pasivo que absorbe el input que recibe tal como se le presenta, la primera pregunta que se puede plantear es: ¿qué tipo de input o exposición a la lengua se debe ofrecer a los alumnos en los primeros estadios de aprendizaje?

Dado que aprender una lengua extranjera en el contexto escolar, lejos del entorno natural, limita muchísimo las posibilidades de exposición, el profesor debe planificar el proceso de enseñanza de tal manera que el alumno reciba el máximo de exposición a la lengua. A este respecto cabe subrayar que el profesor dispone de diversas fuentes para proveer input a sus alumnos.

En primer lugar el microcosmos de la clase constituye por sí mismo una situación de comunicación natural y auténtica de la que el profesor puede sacar el máximo partido utilizando siempre la lengua que se enseña como vehículo de comunicación. Las transacciones comunicativas que se dan en cualquier clase de Enseñanza Primaria como por ejemplo pasar lista, asignación de tareas a los ayudantes, organización y distribución del material, decoración de la clase, reglas de disciplina, salidas al patio, hábitos de orden y limpieza, etc. se producen también en la clase de lengua extranjera por lo que no existe razón para no llevarlas a cabo en la lengua que se enseña. 
Otra fuente de input considerable puede provenir de la organización de las actividades pedagógicas, como son las instrucciones para realizar las tareas, el tiempo asignado, las reglas de los juegos,etc. Dado que en las clases de Primaria estas situaciones se dan frecuentemente, la cantidad de exposición a la larga puede aumentar de manera considerable. Por otro lado la familiaridad de los alumnos con estas situaciones que se producen en el ahora y aquí, el apoyo del contexto y la posibilidad de repetición natural las hacen altamente recomendables.

La tercera fuente de exposición proviene de los contenidos linguiísticos de las múltiples actividades pedagógicas que ofrece el libro de texto o que el mismo profesor diseña para la enseñanza de la lengua como puedan ser las actividades controladas, las rimas, canciones, historias, diálogos, juegos, etc.

Ahora bien, facilitar el máximo de input no sirve para nada si el aprendiz no está predispuesto a recibirlo. Dos condiciones deben darse para que el alumno esté motivado a escuchar: interés y comprensibilidad.

Para promover su interés el profesor ha de llenar los mensajes de contenidos que provoquen la curiosidad de los alumnos. La vida cotidiana real de los alumnos, e incluso la del mismo profesor, fuera y dentro de la escuela ofrece un sinfín de situaciones motivadoras ya que despiertan su curiosidad (rutina diaria, gustos, familia, vacaciones, tiempo libre, etc). La organización de actividades que incluyan elementos lúdicos como los juegos, adivinanzas, etc., o artístico/creativos como el dibujo, trabajos manuales, la música o el teatro y la mismas actividades de organización de la clase pueden llenar de sentido la lengua que el alumno recibe y despertar, por tanto, su interés.

Pero la motivación y el interés nacen también de las posibilidades de comprensión del mensaje. El papel del profesor es aquí insustituible, ya que debe ofrecer a los alumnos que están dando los primeros pasos en el aprendizaje de una lengua un input que sea comprensible y para ello debe utilizar todos los medios posibles facilitadores de la comprensión, como la selección de tópicos y situaciones relevantes para el niño, una pronunciación clara, una entonación significativa, ajustes lingüísticos como la simplificación de la sintaxis o la utilización de léxico de campo semántico amplio, los gestos, el uso del contexto, soportes visuales, así como acomodaciones interaccionales tales como repeticiones, expansiones, etc.

Si la provisión de un input comprensible es fundamental para desencadenar el proceso de adquisición de una lengua, la provisión de situaciones en que el alumno pueda ejercitar su interlengua es también fundamental. El concepto de interlengua como se ha dicho, sostiene que el aprendiz formula sus propias hipótesis, que somete a un proceso de verificación y reformulación en el curso de su desarrollo lingüístico sobre la base del intake que recibe. El profesor, por tanto, debe ofrecer en el contexto del aula oportunidades para que los aprendices puedan acceder libremente a su sistema lingüístico y utilizarlo creativamente y facilidades para que puedan formular y verificar sus propias hipótesis. Ello implicará decisiones por parte del profesor relativas a la planificación y diseño de actividades que favorezcan la producción creativa de lenguaje.

Estas decisiones no conllevan la eliminación de las actividades controladas ya que en una situación de aprendizaje/enseñanza de una lengua extranjera y, especialmente en sus inicios, ofrecen varias ventajas: a) permiten al profesor estructurar su programación, b) constituyen una fuente de input comprensible para el alumno c) permiten al alumno usar la lengua cuando sus recursos interiorizados son aún muy limitados, lo que puede traducirse en una sensación de logro y satisfacción imprescindible para el modelaje de actitudes positivas hacia la lengua y su aprendizaje.

En consecuencia, recursos tales como la memorización de rimas y canciones, al igual que las actividades lingüísticamente controladas, siempre que eviten la repeti- 
ción mecánica y sin sentido de la lengua, tienen su lugar en la clase de lengua extranjera. Lo que el profesor no debe olvidar es que las actividades lingüísticamente creativas, que fomentan la producción de la interlengua de los aprendices deben tener también su lugar en la clase de lengua extranjera, y no evitarlas, como suele ocurrir en los primeros cursos. Estas actividades pueden ser tan simples como algunos juegos de vocabulario en que los aprendices deben recuperar palabras del léxico adquirido, participar en situaciones interactivas próximas a su experiencia, describir ilustraciones o narrar historias muy sencillas sin el recurso a estructuras modelo previamente suministradas.

Es obvio que el profesor debe modelar su actitud ante la producción lingüística del alumno. No es lo mismo valorar una actividad lingüística controlada que otra que estimule la creación linguiística libre. En una actividad controlada los errores pueden tener su origen en la falta de atención o en la incomprensión de cómo llevar a cabo la tarea. Hay que señalar que en ocasiones se puede producir un desacoplamiento entre profesor y alumno. Una actividad que el profesor planifica como controlada, (espera que la producción del alumno se ajuste a las estructuras modelo) puede ser interpretada como libre por el alumno, recurriendo a su propio sistema lingüístico y no a los modelos que se le suministran. Es natural que la producción lingüística del alumno en actividades que fomenten la expresión lingüística espontánea y libre presente desviaciones respecto al sistema linguístico de la lengua objeto de aprendizaje. Estas no deben ser objeto, sin embargo, de evaluación formal, sino comunicativa, o sea valorar la capacidad del alumno para hacerse entender. El profesor por tanto no debe hacer demasiado caso de los errores ni intentar sistemáticamente corregirlos, sino focalizar su esfuerzo didáctico sobre la estimulación de la producción y la reformulación indirecta de las formas inapropiadas del alumno de una forma que puedan servir de modelo a interiorizar.

\section{EL APRENDIZAJE DE LA LENGUA EXTRANJERA EN EL DISEÑO CURRICULAR BASE}

Parece que esta vez la Reforma Educativa ha comprendido la naturaleza y características del proceso de adquisición de una lengua extranjera y así lo ha reflejado en los apartados correspondientes al área de Lenguas Extranjeras de las Disposiciones Oficiales por las que se establecen las enseñanzas mínimas correspondientes a la Educación Primaria y Secundaria (REAL DECRETO 1006/1991, 14 Junio, B.O.E núm.152, de 26 Junio 1991 en las que se explicita de forma clara el objetivo a conseguir (aprender la lengua extranjera para comunicarse) y el papel activo del alumno en la consecución del mismo a través de su propia actuación:

«..la finalidad curricular de esta área no es enseñar una lengua extranjera, sino enseñar a comunicarse en ella..... a través de la puesta en práctica se pone de manifiesto la competencia comunicativa. La actuación del alumno constituye así el núcleo principal de interés de un diseño curricular de lenguas extranjeras» (p. 64, E.P.;p. 119. E.S.)

No se trata como antaño de «enseñar» una competencia gramatical sino de desarrollar una competencia para poder comunicarse que se construirá a través de la propia actuación del alumno; ello significa que el aprendiz debe utilizar la lengua activamente y de manera productiva. El desarrollo de esta competencia comporta un aprendizaje de tipo lingüístico que el Diseño Curricular explicita de la siguiente manera:

«El proceso de adquisición de una lengua extranjera puede caracterizarse como de construcción creativa donde el alumno, apoyándose en un conjunto de estrategias 
naturales, a partir de todo el caudal lingüístico recibido, formula hipótesis para elaborar las reglas que configuran la representación interna del nuevo sistema. ........

La progresión en el aprendizaje no se produce de una forma lineal, sino más bien global, lo que implica necesariamente al principio una simplificación y exclusión de la particularidades que no son percibidas como esenciales....

En un proceso de aprendizaje de esta naturaleza, los errores que comete el alumno ya no pueden ser vistos esencialmente como fallos, sino como la evidencia del dinamismo subyacente en la comprensión y en el dominio progresivos del nuevo sistema comunicativo...) (p. 65,E.P.; p.119, E.S.)

En consecuencia el profesor no sólo debe estar preparado para diseñar actividades para fomentar el uso de la lengua en situaciones comunicativas sino que ha de modelar sus actitudes hacia la actuación de sus alumnos en base a la consideración de los procesos del aprendizaje lingüístico. Por ello y muy especialmente en los estadios iniciales de aprendizaje durante la Educación Primaria el profesor debe ser consciente de las expectativas sobre los niveles de comprensión y producción linguiística de sus alumnos.

Durante los cursos de Primaria y especialmente durante el período de iniciación a la lengua extranjera en el Segundo Ciclo las expectativas del profesor por lo que se refiere al alumno se centraran más en la comprensión que en la producción. La existencia de un período silencioso más o menos largo según los individuos, y que se da en la mayoría de aprendices de una nueva lengua fundamenta esta posición. Ello no significa que el alumno no sea capaz de producir en la nueva lengua sino que esta producción inicial de los alumnos será necesariamente muy rudimentaria, básicamente consistente en palabras aisladas y frases memorizadas. Gradualmente en la medida que el alumno va interiorizando elementos del input que recibe elaborando sus propias hipótesis, empieza a manifestar, sobre todo si el profesor la estimula, una producción linguística creativa en el sentido que no se trata de una mera reproducción memorística sino de emisiones lingüísticas que él mismo construye.

Para terminar queremos insistir en que unos conocimientos básicos a propósito del proceso de adquisición de una lengua extranjera son indispensables para el profesor. Este conocimiento puede ayudar a evitar el sentimiento de frustración que muchos profesores experimentan y que, en el peor de los casos, podría llegar a proyectarse en los alumnos generando en ellos una actitud negativa hacia la lengua y su aprendizaje.

\section{Referencias}

ADJEMIAN, C. (1976). On the nature of interlanguage systems. Language Learning 26, 297-320.

CORDER, S. (1971). Idiosyncratic dialects and error analysis. IRAL, IX, 149-159.

CORDER, S. (1978). Language-learner language. En Richards (ed.), Understanding serond and foreign language learming. Rowley, Mass: Newbury House.

DULAY, H., y BURT, M. (1977). Remarks on creativity in second language acquisition. En M. Burt, H. Dulay y M. Finocchiaro (Eds.). Vieupoints on English as a Serond Language. Nueva York: Regents.

Euws, R. (1982). Discourse Processes in Classroom Serond Language Development. Unpublished doctoral thesis: University of London.

ELLIS, R. (1984). Classroom second language development. Oxford: Pergamon.

EuLS, R. (1985). Understanding Second Language Acquisition. Oxford: O.U.P.

FELIX, S. W. (1981). The effect of formal instruction on second language acquisition. Language Learning, $31,1,87-112$.

FeLIX, S. W., y HAHN, A. (1984). Natural processes in classroom second-language learning. Applied Linguistics, Vol. 6, 3, 223-238.

FeLIX, S. W., y SIMMET, A. (1981). Natural procedures in classroom L2 learning. Revised version of a paper presented at the IIIème Colloque Groupe de Recherche sur l'Acquisition des Langues. París, May 15-17.

HAVRANEK, G. (1988). Das Verbalsystem in der Lemersprache. Tübingen: Gunter Narr Verlag. 
HavraneK, G. (1989). Watching the learner language evolve: The developing verbal system. En G. Willems y P. Riley (eds.), Foreign language learning and teaching in Europe. Amsterdam: Free University Press.

HutCHISON, T. (1985). Project 1. Oxford: O.U.P.

MCNEIL, D. (1966). Developmental psycholinguistics. En F. Smith y G. Miller (Eds.). The Genesis of Language: A Psycholinguistic Approach. Cambridige, Mass.: MIT Press.

MCNEחL, D. (1970). The Acquisition of Language: The Study of Developmental Psycholinguistics. Nueva York: Harper and Row.

MாON, J. (1974). The development of negation in English by a second language learner. TESOL Quarterly 8, 137-143.

NEMSER, W. (1971). Approximative systems of foreign language learners. IRAL, 9, 115-123.

PAVESI, M. (1986). Markedness, discoursal modes and relative clause formation in a formal and an informal context. Studies in Second Language Acquisition, $8(1)$ : 38-55.

RAVEM, R. (1974). The development of Wh-questions in first and second language learners. En J. Richards (Ed.), Error analysis. Londres: Longman.

SELINKER, L. (1972). Interlanguage. IRAL X, 209-230.

TORRAS, M. R. (1992). Assoliment d'objectius didàctics en l'ensenyament de la llengua Anglesa al final de l'EGB (Anälisi de la interllengua dels escolars). Tesi doctoral: Universitat de Barcelona.

TORRAs, M. R. (1993). Un estudio transversal del nivel de adquisición de la interrogación en inglés con alumnos de 8..$^{\circ}$ EGB. Revista Española de Linguḯstica Aplicada. Vol. 9, 169-188.

WODE, H. (1976). Developmental sequences in naturalistic second language acquisition. Working Papers on Bilingualism, 15, 37-57.

WODE, H. (1980). Learning a Second Language. An integrated view of Language Acquisition. Tübingen: Gunter Narr Verlag.

\section{Anexo}

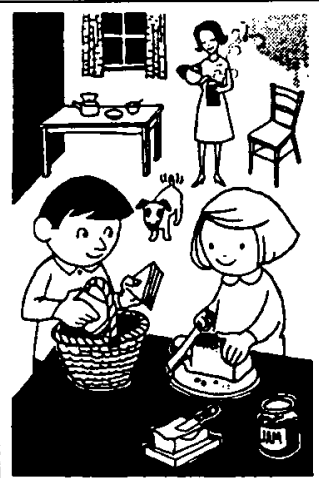

1

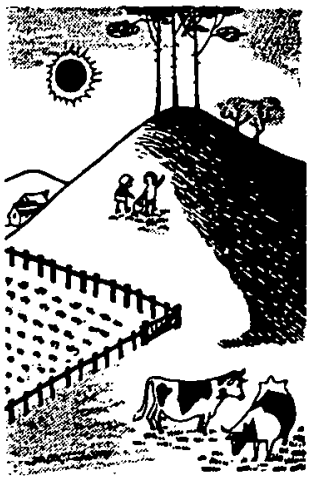

4

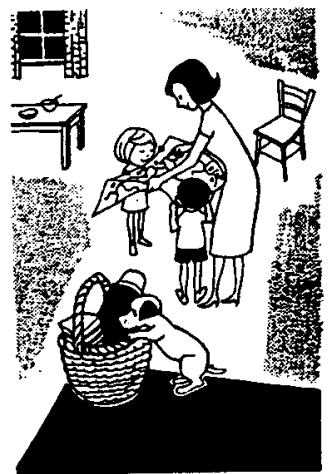

2

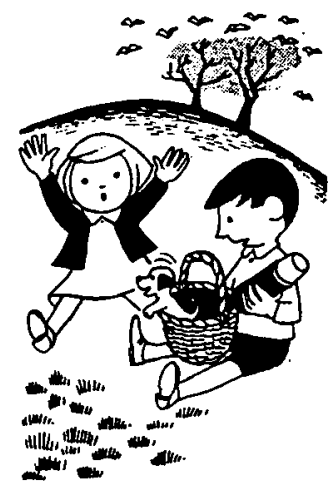

5

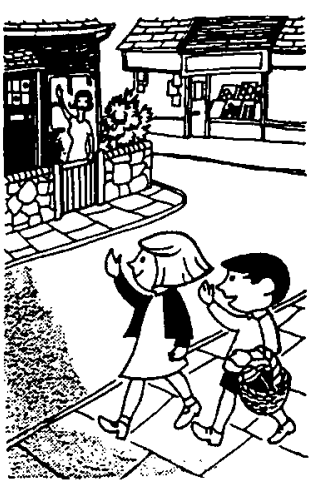

3

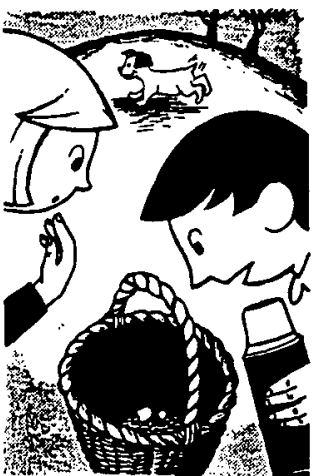

6 
La interlengua en los primeros estadios de aprendizaje de una lengua extranjera (inglés)

M. Rosa Torras i Cherta

$C L \& E, 1994,24, p p .49-62$

Resumen: Las peculiaridades del proceso de adquisición de una L2/LE ponen de manifiesto una serie de estadios o fases a través de los cuales el aprendiz va construyendo de manera global un sistema aproximativo a la lengua objeto de aprendizaje, que constituye por sí mismo un sistema lingüístico en estado continuo de crecimiento y fluctuación, llamado interlengua

En este artículo se analizan las características de la interlengua de los alumnos de $8^{\circ}$ de EGB en relación al proceso de adquisición/aprendizaje del Inglés como LE en un contexto escolar. Partiendo de estos conocimientos se apuntan una serie de implicaciones didácticas orientativas para los profesores que imparten la enseñanza de la LE en el período inicial de su introducción en la Escuela Primaria.

Dirección: Departament de Didàctica de la Llengua i la Literatura. Universitat de Barcelona Melcior de Palau, 140, 08014 - Barcelona

(C) PERMISOS PARA CITAR O REPRODUCIR EN OTRAS FUENTES: Se pueden citar libremente hasta 500 palabras. Para reproducir una porción de texto mayor, figuras o ilustraciones, se deberá pedir permiso por escrito a la revista, especificando el uso al que se destina el texto. En todos los casos, se deberá citar el copyright de $C L \& E$. En el caso de artículos o textos que hayan sido a su vez reproducidos en $C L \& E$ los interesados deberán dirigirse tanto a los detentadores del copyright original como a $C L \& E$, en el caso de que se quiera hacer uso de la traducción. FOTOCOPIAS: Para todo lo relacionado con el uso mediante fotocopia del material de esta revista, deberán dirigirse a: CEDRO, C/ José Marañón, 10,3. Izda. Tel. 5941575 . Fax 4453567 